Article

\title{
Evaluation of the Use of Blood Products in ICU Hospitalized COVID-19 Patients
}

\author{
Branislava Vasiljević-Jovanović ${ }^{1, *}$, Marija Milenković ${ }^{2,3}{ }^{\oplus}$, Lidija Mijović $^{4}$, Zoran Bukumirić $^{5}$, \\ Milena Šantrić-Milićević ${ }^{6}{ }^{\infty}$, Miloš Božanić ${ }^{7}$, Vojislava Milutinović ${ }^{8}$ and Marija Zdravkovićc ${ }^{3,9}$
}

check for

updates

Citation: Vasiljević-Jovanović, B.; Milenković, M.; Mijović, L.;

Bukumirić, Z.; Šantrić-Milićević, M.;

Božanić, M.; Milutinović, V.;

Zdravković, M. Evaluation of the Use of Blood Products in ICU

Hospitalized COVID-19 Patients.

Surgeries 2021, 2, 391-398. https://

doi.org/10.3390/surgeries2040039

Academic Editor: Sibylle Kietaibl

Received: 27 September 2021

Accepted: 17 November 2021

Published: 20 November 2021

Publisher's Note: MDPI stays neutral with regard to jurisdictional claims in published maps and institutional affiliations.

Copyright: (c) 2021 by the authors. Licensee MDPI, Basel, Switzerland. This article is an open access article distributed under the terms and conditions of the Creative Commons Attribution (CC BY) license (https:// creativecommons.org/licenses/by/ $4.0 /)$.
1 Blood Bank Department, University Hospital Medical Center Bezanijska kosa, 11080 Belgrade, Serbia

2 Department of Anaesthesiology, Clinical Center of Serbia, 11000 Belgrade, Serbia; smgk055@gmail.com

3 Faculty of Medicine, University of Belgrade, 11000 Belgrade, Serbia; sekcija.kardioloska@gmail.com

4 Department of Clinical Transfusion Medicine, Blood Transfusion Institute of Serbia, 11000 Belgrade, Serbia; lidijamijovicnikolic@gmail.com

5 Institute for Medical Statistics and Informatics, Faculty of Medicine, University of Belgrade, 11000 Belgrade, Serbia; zoran.bukumiric@med.bg.ac.rs

6 Institute for Social Medicine, Faculty of Medicine, University of Belgrade, 11000 Belgrade, Serbia; milena.santric-milicevic@med.bg.ac.rs

7 Department of Medical Informatics, University Hospital Medical Center Bezanijska kosa, 11080 Belgrade, Serbia; bozanic.milos@bkosa.edu.rs

8 Department of Emergency Medicine, University Hospital Medical Center Bezanijska kosa, 11080 Belgrade, Serbia; mvojislava@gmail.com

9 Department of Cardiology, University Hospital Medical Center Bezanijska kosa, 11080 Belgrade, Serbia

* Correspondence: bvjovanovic@yahoo.com

Abstract: (1) Background: The COVID-19 pandemic tested the public health system's readiness for crises and highlighted the importance of knowing the demand for blood products and the maintenance of the blood supply chain. The aim of this study was to evaluate blood product usage in a series of patients that were hospitalized due to COVID-19 and to analyze their demographics and clinical characteristics. (2) Methods: In this retrospective cohort study, we analyzed data from transfused COVID-19 patients that were treated in the University Hospital Medical Center Bezanijska Kosa in Belgrade, Serbia during the second wave of the epidemic. (3) Results: This study included 90 patients. The median age of the patients was 72 (range 23-95) years. The median time of hospitalization was 23 days (range 3-73 days). In intensive care units (ICUs) the median time of hospitalization was 9 days (range 0-73). One or more comorbidities were observed in 86 individuals (95.6\%). The total number of transfused red blood cell concetrates (RBC) was 304 (139 in ICU, 165 in other wards), with a mean of 3 units/patient (range 1-14). Comorbidities, severity of illness and hospital duration in the ICU were statistically significant predictors of higher RBC use. (4) Conclusion: Knowledge of the transfusion profile of COVID-19 patients allowed better management of the hospital's blood stocks during the COVID-19 pandemic.

Keywords: COVID-19; blood transfusion; RBC; ICU; blood supply

\section{Introduction}

Currently, in the second year of the COVID-19 pandemic, we are still learning about the different consequences of the disease. [1]. The epidemiological and clinical characteristics, and diagnostic tools, have been comprehensively investigated worldwide and have been presented in many studies. It is estimated that $20-55 \%$ of patients with COVID-19 have coagulopathy [2]. The use of blood products in patients hospitalized with COVID-19 also needs to be better investigated. Currently, data in the literature on the need of blood transfusion in these patients are scarce [3]. Studies suggest that red blood cell concentrate (RBC) is the component most often transfused in COVID-19 patients in ICUs. The use of other blood products, such as platelet concentrates, fresh frozen plasma (FFP) and 
cryoprecipitate (cryo) is low. It has also been suggested that the unnecessary transfusion of plasma components should be avoided in patients who do not have major, active bleeding [4]. Anaemia in SARS-CoV-2 infection seems to be correlated with severity of illness as a result of multiple pathogenetic mechanisms [5]. The restrictive blood transfusion strategy suggests a transfusion trigger of a hemoglobin level $<70 \mathrm{~g} / \mathrm{L}$ for critically ill patients. Available data do not provide us with sufficient information about whether patients with considerable respiratory symptoms, who need respiratory support, may benefit from RBC transfusions to maintain hemoglobin levels above $70 \mathrm{~g} / \mathrm{L}$ [6]. Patients with COVID-19 are often elderly with pre-existing chronic conditions. In current guidelines, the hemoglobin level is to date the most commonly used trigger for RBC administration for the correction of anemia. However, hemoglobin level is not the only parameter to be considered when deciding on potential RBC transfusion [7]. It has also been suggested that patients in ICUs require more transfusions. Rim et al. found that the prevalence of the need for blood transfusion was $78.5 \%$ in ICUs [8]. Transfusion-related acute lung injury (TRALI) and transfusion-associated circulatory overload (TACO) are adverse transfusion reactions, which can intensify COVID-19-associated acute respiratory distress syndrome (ARDS) [9]. Considering other side effects, such as transfusion-related immunomodulation (TRIM), a careful individual balancing of risk and benefit is mandatory. As long as specific transfusion recommendation for patients with COVID-19 are not fully developed, physicians should follow general recommendations to transfuse these patients [10]. In light of all the above, a need arises for hospitals to support the implementation of patient blood management (PBM) principles for optimizing hemostasis and hemoglobin levels, as well as the use of blood products and blood alternatives [11].

The COVID-19 pandemic tested the public health system's readiness for crises. During the pandemic, the hospital blood transfusion service also faced a number of challenges related to the voluntary and safe collection of blood. The challenge to maintain adequate blood supplies arose because of the reduced availability of blood components due to decreased donations. Worldwide, it was, and still is, necessary to implement additional strategies to attract donors, who were often in social isolation and working from home [12]. In this regard, knowledge of the demand for blood, plasma and platelets is essential for the promotion and maintenance of the blood supply chain, as well as for efficient and equitable distribution in accordance with the requirements of hospital patients to be adequately supplied [13]. According to the Serbian Ministry of Health recommendation, hospitals included in the system of "COVID-19 institutions" had priority for blood supply. Blood shortages here, as in other countries, were often avoided. However, at some point the equilibrium in the blood banks shifted from donation to transfusion although the elective surgery and blood usage was dramatically reduced. The important quality indicator of appropriate blood usage for the hospital, and the communication between the clinician and the blood center, is the crossmatch/transfusion $(\mathrm{C} / \mathrm{T})$ ratio. The $\mathrm{C} / \mathrm{T}$ ratio is the total number of units crossmatched divided by the total units actually transfused. Unnecessary blood requests lead to the loss of stock in the blood centers and increases costs associated with its preparation and crossmatching of the blood components [14].

The aim of this study is to evaluate the usage of blood products in a series of adult patients that were hospitalized due to COVID-19 in the ICU of University Hospital Medical Center Bezanijska Kosa, Belgrade (Serbia) and to analyze their demographics and clinical characteristics. Knowledge about the predictors of the need for blood transfusions could inform guidelines to optimize resources of blood products in the most appropriate way during blood shortages.

\section{Materials and Methods}

The study was a retrospective cohort study, which included transfused COVID-19 patients treated in the Clinical Hospital Center "Bezanijska Kosa" in Belgrade, Serbia between 23 June 2020 and 2 October 2020 during the second wave of the COVID-19 pandemic in Serbia. Throughout the study period, the hospital was exclusively a COVID- 
19 hospital. The study was approved by the Ethics Committee of University Hospital Medical Center Bezanijska Kosa, Belgrade, Serbia (5425/1/), 16.08.2021. All patients with a verified diagnosis of the COVID-19 disease (confirmed by real-time PCR assay), patients over 18 years of age, patients who met the illness severity criteria for stage three or higher (according to the COVID-19 National Guidelines, version nine) and were receiving blood and blood products transfusion were the criteria for inclusion in the study. Variables of interest included demographic, health status and health care variables. Criteria for excluding patients from the study were incomplete data and the transfer of the patient to another medical institution for COVID-19 treatment. The primary outcome was defined as the number of transfused blood components during the whole stay.

According to the decision of the Ministry of Health from 23 June 2020, the hospital was included in the system of "COVID-19 institutions of Serbia" and all the time during the study period this hospital, with all 360 beds, was included in the system and had a priority status in the blood chain supply. The blood was mainly supplied from the National Blood Institute of Serbia and additionally from another clinical hospital center. Infection with SARS-CoV-2 virus was confirmed by RT-PCR assay according to WHO guidelines [15]. A chest CT was obligatory at admission, including the estimation of CT severity score. Patients were treated according to the adjusted National Protocol of the Republic of Serbia for the treatment of COVID-19 infection, which included anticoagulants in standard prophylactic or therapeutic doses among other applied therapies. The indication for convalescent plasma was established by a specific scoring system according to the same national protocol [16]. Illness severity was defined by the WHO Clinical Management of COVID-19 guidance. Patients were treated by a multidisciplinary team, which consisted of intensivists, internists, radiologists and surgeons.

The necessary data were obtained from medical documentation and the health information system of the University Hospital Medical Center Bezanijska Kosa (Heliant, v7.3, r48602) and from the blood bank protocols. The electronic database included demographic data (age, gender), laboratory values (Hgb), hospital days, type of hospital unit, respiratory support days and the outcome of the treatment (in-hospital, mortality/discharge). Other recorded data included past medical history (cardiovascular disease and hypertension, diabetes mellitus, chronic obstructive pulmonary disease (COPD) and asthma bronchiale, chronic kidney disease, neurological and neuromuscular chronic diseases, immunodeficiencies and HIV, cancer, chronic liver disease (CLD), chest CT severity score and anticoagulant therapy). All data regarding the pretransfusion testing (blood group and compatibility tests) and blood transfusions were collected from the blood bank protocols and blood request forms. We identified all blood products issued in this period: red blood cells, platelet concentrates, fresh frozen plasma, convalescent plasma and cryoprecipitate. The $\mathrm{C} / \mathrm{T}$ ratio in the relevant period was calculated using the following equation: [Crossmatch to transfusion ratio $=$ Number of units crossmatched/number of units transfused]. International standards of blood centers recommend a $\mathrm{C} / \mathrm{T}$ ratio $<2$ [17].

Statistical analysis: Results were presented as frequency (percent), median (range) and mean $\pm \mathrm{sd}$. For the modeling of the relation of the dependent variable (number of RBC units) with potential predictors, a quantile regression was used. All $p$-values less than 0.05 were considered significant. Statistical data analysis was performed using IBM SPSS Statistics 22 (IBM Corporation, Armonk, NY, USA).

\section{Results}

Throughout the research period 1523 patients with COVID-19 were treated in this hospital and 90 were transfused (5.9\%). Table 1 shows the baseline clinical and transfusion laboratory data of patients included in the study. Out of all 90 patients, $50(55.6 \%)$ were male and $40(44.4 \%)$ females. The median age of patients was 72 (range 23-95) years. The median time of hospitalization was 23 days (range 3-73 days). In intensive care units (ICUs) the median time of hospitalization was 9 days (range 0-73). The median days of invasive respiratory support for patients who presented with respiratory distress was 0.50 days 
(range 0-72). A total of 36 patients were admitted to intensive care units (40.0\%) and 54 to other wards (60.0\%). The median chest CT severity score was 20.0 (range 4-25). During hospitalization anticoagulant therapy was used for 80 patients $(88.9 \%)$. A total of $39(43.3 \%)$ patients with SARS-CoV-2 infection were categorized as moderately severe and 51 (56.7\%) as severe/critical in severity of illness categories. During the treatment $36(40 \%)$ patients died and $54(60 \%)$ patients were discharged from the treatment (Table 1).

Table 1. Baseline and transfusion data of the patients.

\begin{tabular}{|c|c|}
\hline Variables & All Patients $n=90$ \\
\hline Age, median (range) & $72(23-95)$ \\
\hline Gender, male/female, $n(\%)$ & $50(55.6) / 40(44.4)$ \\
\hline Pts in ICU/ward, $n(\%)$ & $36(40) / 54(60)$ \\
\hline Hospital days, median (range) & $23(3-73)$ \\
\hline Hospital days in ICU, median (range) & $9(0-73)$ \\
\hline Invasive respiratory support days, median (range) & $0.5(0-72)$ \\
\hline Chest CT severity score, median (range) & $20(4-25)$ \\
\hline All comorbidities, $n(\%)$ & $86(95.6)$ \\
\hline Cardiovascular/HTA, $n(\%)$ & $71(78.9 \%)$ \\
\hline Neurological/neuromuscular & $17(18.9 \%)$ \\
\hline $\mathrm{DM}, n(\%)$ & $24(26.7 \%)$ \\
\hline COPD/asthma, $n(\%)$ & $11(12.2 \%)$ \\
\hline Immunodeficiency/HIV, $n(\%)$ & $6(6.7 \%)$ \\
\hline Cancer, $n(\%)$ & $18(20.0 \%)$ \\
\hline CLD, $n(\%)$ & $3(3.3 \%)$ \\
\hline $\mathrm{CKD}, n(\%)$ & $18(20.0 \%)$ \\
\hline Anemic pts. at admission, $n(\%)$ & $50 / 90(55.6)$ \\
\hline Transfused RBC/Plt/FFP/Cryo, n & $304 / 42 / 96 / 58$ \\
\hline Transfused RBC ICU/ward in total, $n(\%)$ & $139(45.7 \%) / 165(54.3 \%)$ \\
\hline \multicolumn{2}{|l|}{ Transfused units per patient, median (range): } \\
\hline RBC & $3(1-14)$ \\
\hline Plt & $1(1-7)$ \\
\hline FFP & $3.5(1-16)$ \\
\hline \multirow{2}{*}{$\begin{array}{c}\text { Cryo } \\
\text { Hemoglobin at transfusion, g/dL ICU/ward, median } \\
\text { (range) }\end{array}$} & $10(6-12)$ \\
\hline & $75(59-121) / 77$ (53-96) \\
\hline In-hospital mortality $n(\%)$ & $36(40.0 \%)$ \\
\hline
\end{tabular}

Categorical variables are expressed as numbers and percentages; continuous variables are expressed as the mean ( \pm standard deviation) or median (IQR). ICU: intensive care unit; RBC: red blood cells; Plt: platelets; FFP: fresh frozen plasma; Cryo: cryoprecipitate.

Clinical comorbidities were analyzed in all patients. One or more comorbidities were observed in 86 individuals (95.6\%). The median number of comorbidities was 2 (range 0-6). The most common were arterial hypertension and other cardiovascular diseases which were recorded in 71 subjects (78.9\%), followed by diabetes mellitus in 24 subjects (26.7\%), cancer in 18 patients $(20.0 \%)$, chronic kidney disease also in 18 patients $(20.0 \%)$, chronic neurological and neuromuscular diseases in 17 patients $(18.9 \%)$, COPD and asthma bronchiale in 11 patients $(12.2 \%)$, immunodeficiency including HIV in 6 patients $(6.7 \%)$, chronic liver disease in 3 patients (3.3\%), while other comorbidities were less common. Quantile regression with the number of $\mathrm{RBC}$ doses as a dependent variable $(\mathrm{N}=90)$ is shown in Table 2.

Statistically significant variables from univariate analyses were included in the multivariate regression model with the number of RBC doses as a dependent variable. Due to multicollinearity with the variable number of days of hospitalization, and the department of variables, the number of days in the ICU was not included in the multivariate model. In the multivariate model, a statistically significant predictor of higher RBC dose count is a higher number of comorbidities $(\mathrm{B}=0.847 ; p=0.009)$. 
Table 2. Quantile regression with the number of RBC doses as a dependent variable, $\mathrm{N}=90$.

\begin{tabular}{ccccc}
\hline \multirow{2}{*}{ Variable } & \multicolumn{2}{c}{ Univariate } & \multicolumn{2}{c}{ Multivariate } \\
\cline { 2 - 5 } & B & $p$-Value & B & $p$-Value \\
\hline $\begin{array}{c}\text { Gender } \\
\text { (m vs. f) }\end{array}$ & $<0.001$ & 1.000 & & \\
\hline Age (years) & $<0.001$ & 1.000 & & \\
\hline Total hospital days & 0.059 & 0.010 & 0.041 & 0.071 \\
\hline Hospital days in the ICU & 0.054 & 0.023 & & \\
\hline Respiratory support days & 0.047 & 0.093 & & \\
\hline Place of residence (Belgrade vs. others) & $<0.001$ & 1.000 & & \\
\hline Ward (ICU vs. others) & 2.000 & 0.002 & -0.143 & 0.874 \\
\hline Chest CT severity score & -0.050 & 0.570 & & \\
\hline Comorbidities & 1.000 & 0.002 & 0.847 & 0.009 \\
\hline Anticoagulant therapy & -1.000 & 0.434 & & \\
\hline Illness severity (moderately severe vs. severe) & 2.000 & 0.001 & 1.296 & 0.173 \\
\hline
\end{tabular}

In total, 500 blood products were administered including packed red blood cells (RBC), platelet concentrates and apheresis platelet concentrate (Plt), fresh frozen plasma (FFP) and units of cryoprecipitate (cryo) into the 90 patients. A total of 50 patients $(55.6 \%$ ) had pre-existing anemia. Furthermore, $57 \%$ received one to three RBC units and $43 \%$ four or more. A total of 304 RBCs (139 in ICU and 165 in other wards) were transfused, with a mean of 3 units/patient (range 1-14). In addition, a total of 42 platelet units were transfused in five patients with a mean of 1 (range 1-7), 96 FFPs were used in 15 patients, with a mean of 3.5 (range 1-16), and 58 cryo were used in 6 patients, with a mean of 10 (range 6-12). The mean hemoglobin level (Hgb) at transfusion was $75 \mathrm{~g} / \mathrm{L}$ (range 59-121 g/L) in the ICU $\left(\mathrm{N}_{\mathrm{RBC}}=139\right)$ and $77 \mathrm{~g} / \mathrm{L}$ (range 53-96 g/L) in other wards $\left(\mathrm{N}_{\mathrm{RBC}}=165\right)$ (Figure 1). Four patients received convalescent plasma. Among the 90 SARS-CoV-2 patients the blood group percentage distribution was $52.2 \%, 20.0 \%, 22.2 \%$ and $5.6 \%$ for $\mathrm{A}, \mathrm{B}, \mathrm{O}$ and $\mathrm{AB}$, respectively. The Rh D blood group percentage distribution was $88.9 \%$ positive and $11.1 \%$ negative. All transfused components were $\mathrm{ABO}$ and $\mathrm{Rh} \mathrm{D}$ identical. No transfusion reactions or adverse events were reported. The number of crossmatch tests performed was 378 and the number of RBCs issued 304. The $\mathrm{C} / \mathrm{T}$ ratio for our hospital during the study period was 1.2, which is considered indicative of optimum blood usage.

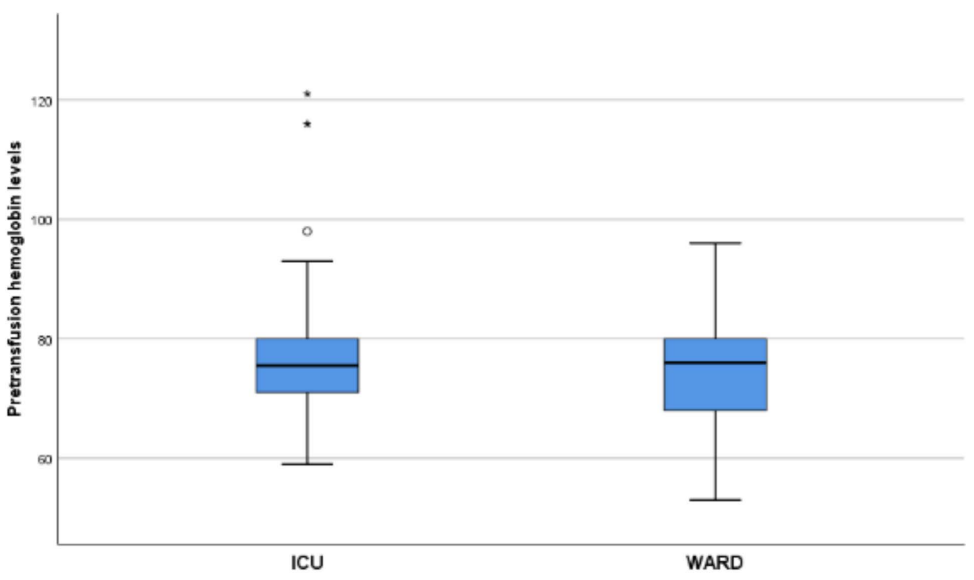

Figure 1. The mean hemoglobin level $(\mathrm{Hgb})$ at transfusion was $75 \mathrm{~g} / \mathrm{L}$ (range 59-121g/L) in the ICU $(\mathrm{NRBC}=139)$ and $77 \mathrm{~g} / \mathrm{L}$ (range 53-96 g/L) in other wards $(\mathrm{NRBC}=165)$. NRBC: number of red blood cell concetrates. 


\section{Discussion}

The most important findings of this study are as follows: the need for more transfusion is significantly higher in patients with longer hospitalization and more underlying illnesses, and that patients who are admitted to ICUs have higher rates of red blood cell transfusion than those admitted to other wards. This study indicated comorbidities as the main predictor of the number of required RBC doses. Dalmazzo et al. also found that the need for transfusion was significantly higher in patients admitted to ICUs. Their study, which included a large number of patients, also found that COVID-19 patients had low rates of blood utilization and that with the consequent suspension of elective surgeries, the number of transfusions decreased in general [3]. We observed in our study that $55.6 \%$ were anemic at admission. Other authors found that pre-existing anemia, and specifically anemia of inflammation, was frequent in patients with severe SARS-CoV-2 infection and was corelated to a longer duration of hospital treatment and poor outcome. They suggested that the analysis of pre-existing anemia as a risk factor for COVID-19 infection needs to be investigated more [5].

The COVID-19 pandemic aroused many health, economic and social problems in society. The need for blood and blood products depends on the number of inhabitants, number of hospital beds and types of medical services provided in the country. The requirements in Serbia are approximately 40 blood donors per 1000 inhabitants (4\%) [18], which was difficult to achieve even before the COVID-19 crisis. A decline in the number of donations was expected with the start of the pandemic with the government epidemiological measures to control the spread of infection. In the first year of the COVID-19 pandemic, a 20\% decline in blood donations was published by the main national blood establishment [18]. University Hospital Medical Center Bezanijska Kosa is a general hospital, with 360 acute hospital beds allocated for internal medicine, surgery and oncology inpatient care. In the pre-pandemic period, ICU patients were mostly critically ill patients requiring surgery or recovering from surgery. Our hospital was particularly affected by the pandemic by becoming a dedicated 'COVID-19' hospital with a drastic decline or absence of elective surgeries and other medical admissions. Consequently, blood use decreased for indications other than the treatment of COVID-19 cases. In 2019, in the same period of time as in our study, out of the total of 3557 hospitalized patients, 445 (12.5\%) needed transfusion, for which a total of 1187 RBC was issued (of which, $23.5 \%$ in the ICU and $76.5 \%$ in other wards, including the operating theater). Comparing this to $5.9 \%$ of transfused patients in 2020, the number of patients who needed transfusion was halved and total number of RBC doses issued in our hospital was 304 (of which $45.7 \%$ was in the ICU and $54.3 \%$ was in wards, including the operating theater).

The crossmatch to transfusion ratio $(\mathrm{C} / \mathrm{T}$ ratio) is an important quality work indicator in blood banks. The $\mathrm{C} / \mathrm{T}$ ratio of $1.2(378 / 304)$ in 2020 was lower than the $\mathrm{C} / \mathrm{T}$ ratio of $1.6(1807 / 1142)$ reported in the same period of 2019, the year before the COVID-19 pandemic. Both C/T ratios are optimal $(<2)$, while the ratio of 1.0 (all crossmatched blood is transfused) would be ideal. This result indicated that the blood bank performed within the recommended value of $<2$, while a ratio of 1.0 (all crossmatched blood is transfused) would be ideal.

Phenotypic frequencies of $\mathrm{ABO}$ blood groups in the population of Serbia are $42.6 \%$, $18 \%, 30.6 \%, 8.6 \%$ for $\mathrm{A}, \mathrm{B}, \mathrm{O}$ and $\mathrm{AB}$, respectively [19]. The findings of other studies exhibited a considerably higher risk of acquiring SARS-CoV-2 for blood group " $\mathrm{A}$ " individuals and a lower risk for blood group "O" individuals [20]. For severe COVID-19 patients in our hospital, there was a skew towards group A based on the frequencies within the Serbian population.

Strength and Limitations: this study has several limitations. First, it was a single center, retrospective study. In addition, the sample size was relatively small. Laboratory data were not uniformly available for every patient so we did not perform dynamic measurement of hemoglobin level. The clinical indications for transfusion were not available for all patients so we could not investigate the clinical reasons for pretransfusion anemia, other 
than hemoglobin level. In the study period, 100 patients required transfusion, but only 90 of them were included in the study due to insufficient medical records data for analyzing.

\section{Conclusions}

This study indicated comorbidity, severity of illness, admission to ICU and length of hospital stay as drivers for an increased use of red blood cells for hospital treatment of patients with verified a diagnosis of COVID-19 disease. While we await the results of further prospective controlled studies, the evidence from this retrospective and singlehospital study could provide valuable information for planning the requirements for the transfusion treatment in outbreaks.

Author Contributions: Conceptualization, B.V.-J. and L.M.; methodology, B.V.-J. and M.Š.M.; formal analysis and software, Z.B. and B.V.-J.; validation, B.V.-J. and M.Š.M.; investigation, B.V.-J., M.M., M.B. and V.M.; data curation, B.V.-J. and Z.B.; resources, M.Z.; writing-original draft preparation, B.V.-J. All authors have read and agreed to the published version of the manuscript.

Funding: This research received no external funding.

Institutional Review Board Statement: The study was conducted according to the guidelines of the Declaration of Helsinki and approved by the Ethics Committee of University Hospital Medical Center Bezanijska Kosa, Belgrade, Serbia (5425/1/), 16.08.2021.

Data Availability Statement: The data that support the findings of this study are available from the corresponding author upon reasonable request.

Acknowledgments: The authors would like to thank Nick Schofield, whose native language is English, for editing the manuscript. The authors would like to acknowledge all healthcare professionals who are in the front line against COVID-19 disease in the Republic of Serbia and worldwide.

Conflicts of Interest: The authors declare that there is no conflict of interest regarding the publication of this paper.

\section{References}

1. Munster, V.J.; Koopmans, M.; van Doremalen, N.; van Riel, D.; de Wit, E. A Novel Coronavirus Emerging in China-Key Questions for Impact Assessment. N. Engl. J. Med. 2020, 382, 692-694. [CrossRef] [PubMed]

2. Lee, S.G.; Fralick, M.; Sholzberg, M. Coagulopathy associated with COVID-19. CMAJ 2020, 192, E583. [CrossRef] [PubMed]

3. Dalmazzo, L.; de Almendra Freitas, A.F.; Alves, B.E.; Cardoso, D.K.; de Carvalho, E.F.; Akil, F.; da Cunha Vieira Perini, F.; Pires, K.T.; de Aguiar, L.C.; Moraes, M.C.; et al. Transfusion profile, clinical characteristics, comorbidities and outcomes of 3014 hospitalized patients diagnosed with COVID-19 in Brazil. Vox Sanguinis 2021. [CrossRef] [PubMed]

4. Doyle, A.J.; Danaee, A.; Furtado, C.I.; Miller, S.; Maggs, T.; Robinson, S.E.; Retter, A. Blood component use in critical care in patients with COVID-19 infection: A single-centre experience. Br. J. Haematol. 2020, 191, 382-385. [CrossRef] [PubMed]

5. Bellmann-Weiler, R.; Lanser, L.; Barket, R.; Rangger, L.; Schapfl, A.; Schaber, M.; Fritsche, G.; Wöll, E.; Weiss, G. Prevalence and Predictive Value of Anemia and Dysregulated Iron Homeostasis in Patients with COVID-19 Infection. J. Clin. Med. 2020, 9, 2429. [CrossRef] [PubMed]

6. Stanworth, S.J.; New, H.V.; Apelseth, T.O.; Brunskill, S.; Cardigan, R.; Doree, C.; Germain, M.; Goldman, M.; Massey, E.; Prati, D.; et al. Effects of the COVID-19 pandemic on supply and use of blood for transfusion. Lancet Haematol. 2020, 7, e756-e764. [CrossRef]

7. Chandra, S.; Kulkarni, H.; Westphal, M. The Blood mess of red blood cell transfusion. Crit. Care 2017, 21 (Suppl. 3), 310. [CrossRef] [PubMed]

8. Rim, J.H.; Lee, S.A.; Han, C.H.; Yoo, J. Transfusion demand in COVID-19 patients from the Korean population: A nationwide study in South Korea. Br. J. Haematol. 2020, 190, e323-e326. [CrossRef] [PubMed]

9. Baron, D.M.; Franchini, M.; Goobie, S.M.; Javidroozi, M.; Klein, A.A.; Lasocki, S.; Liumbruno, G.M.; Muñoz, M.; Shander, A.; Spahn, D.R.; et al. Patient blood management during the COVID-19 pandemic: A narrative review. Anaesthesia 2020, 75, 1105-1113. [CrossRef] [PubMed]

10. Grandone, E.; Pesavento, R.; Tiscia, G.; De Laurenzo, A.; Ceccato, D.; Sartori, M.T.; Mirabella, L.; Cinnella, G.; Mastroianno, M.; Dalfino, L.; et al. Mortality and Transfusion Requirements in COVID-19 Hospitalized Italian Patients According to Severity of the Disease. J. Clin. Med. 2021, 10, 242. [CrossRef] [PubMed]

11. Shander, A.; Corwin, H.L. A narrative review on hospital-acquired anemia: Keeping blood where it belongs. Transfus. Med. Rev. 2020, 34, 195-199. [CrossRef] [PubMed] 
12. Maintaining a Safe and Adequate Blood Supply during the Pandemic Outbreak of Coronavirus Disease (COVID-19). WHO Interim Guidance. February 2021. Available online: https://www.who.int/publications/i/item/WHO-2019-nCoV-BloodSupply-2021-1 (accessed on 11 September 2021).

13. Mascaretti, L.; De Angelis, V.; Berti, P. The severe acute respiratory syndrome coronavirus 2 (SARS-CoV-2) pandemic and Transfusion Medicine: Reflections from Italy. Blood. Transfus. 2020, 18, 77-78. [PubMed]

14. Lukic, V.; Zivotic, B.; Vasiljevic, B.; Sabani, A.; Bogdanovic, G.; Kovac, M. Rational red blood cells administration-have we achieved a satisfactory level? Srpski Arhiv za Celokupno Lekarstvo 2020, 148, 299-303. [CrossRef]

15. World Health Organization. Laboratory Testing for Coronavirus Disease (COVID-19) in Suspected Human Cases: Interim Guidance. World Health Organization. 19 March 2020. Available online: https://apps.who.int/iris/handle/10665/331501.

16. Popadic, V.; Klasnja, S.; Milic, N.; Rajovic, N.; Aleksic, A.; Milenkovic, M.; Crnokrak, B.; Balint, B.; Todorovic-Balint, M.; Mrda, D.; et al. Predictors of Mortality in Critically Ill COVID-19 Patients Demanding High Oxygen Flow: A Thin Line between Inflammation, Cytokine Storm, and Coagulopathy. Oxidative Med. Cell. Longev. 2021, 6648199. [CrossRef] [PubMed]

17. Thomas, R.A.B.; Daniels, H.; Duncan, J. Blood transfusion in general Surgery, MSBOS guidelines are accurate and can decrease blood wastage. Int. J. Surg. 2010, 8, 559. [CrossRef]

18. National Blood Transfusion Institute of Serbia. Available online: https://www.nbti.org.rs/NBTI/1102/Voluntary-blood-donor. shtml (accessed on 15 September 2021).

19. Vojvodic, S. Distribution of ABO, Rh, MNSs, Kell, and Duffy blood-group antigens in population of Vojvodina. Med. Pregl. 2003, LVI, 173-177. [CrossRef]

20. Wu, Y.; Feng, Z.; Li, P.; Yu, Q. Relationship between ABO blood group distribution and clinical characteristics in patients with COVID-19. Clin. Chim. Acta 2020, 509, 220-223. [CrossRef] [PubMed] 